\title{
APPLYING THE GOTHI MODEL OF TACTILE AND HAPTIC INTERACTIONS
}

\author{
Dr. Keith Nesbitt \\ Charles Sturt University, Australia \\ knesbitt@csu.edu.au \\ Dr. Jim Carter \\ University of Saskatchewan, Canada \\ carter@cs.usask.ca
}

\begin{abstract}
This paper discusses the emerging area of tactile and haptic display and some of the breadth of applications of tactile/haptic interactions. While many research studies have provided ergonomic insights into the design of tactile/haptic interactions, the many dimensions and properties of these interactions make it especially difficult to combine the guidance from these individual studies. The GOTHI-05 workshop (Guidelines on Tactile and Haptic Interactions, October 2005) brought researchers together to develop a collection of ergonomic guidance and a framework (the GOTHI model of tactile and haptic interaction (Carter, van Erp, et. al., 2005)) for organizing this guidance. The inaugural meeting of ISO TC159/SC4/WG9 further refined this framework and adopted it as the basis for structuring its new series ISO standards on tactile and haptic interactions. The model itself will be elaborated in ISO 9241-910 Framework for Tactile and Haptic Interactions. The model has already proven useful in identifying and organizing specific guidelines in the first drafts of ISO 9241-920 Guidance on Tactile and Haptic Interaction.
\end{abstract}

The paper discusses the various dimensions and properties of tactile/haptic interactions, identified in an expanded version of GOTHI model and identifies major considerations based on this model for use by developers (and potentially by evaluators) of interfaces that make use of tactile/haptic interactions ISO, 2006).

\section{INTRODUCTION}

Tactile and Haptic displays have been the subject of research for a number of years and in the case of some niche domains, such as remote manipulation have been used extensively. However, it is only recently that more generic devices have been available at a cost that has allowed them to be used in more standard computing interfaces. For some applications it might be argued that tactile and haptic display is simply an unnecessary modality, with most information being adequately conveyed through visual and auditory channels. However, the tactile/haptic sense is adept at perceiving certain modalities of information that are not readily received through the other senses (Friedes, 1974). As expected, this extra channel of information is important in designing interfaces which are accessible to all users, for example, those with visual impairments.

It should be noted that work in this area is still quite embryonic. The early nature of work in this field is demonstrated by the fact that even the definition of terms like 'Tactile' and 'Haptic' are not agreed on. In this paper we use the word haptics to encompass the full range of sensations associated with manipulation and exploration of objects. Haptic sensation is provided through a number of different senses, and integrates information about forces, spatial position (kinesthetic) and a range of surface (tactile) properties. The word tactile is used in relation to information sensed through contact with the skin. Tactile information is not provided through a single type of receptor but a range of receptors that measures qualities such as flutter, vibration, stretch, pressure, temperature and even chemical properties such as itch. A more complete description of the haptic and tactile sense is provided in the next section.

Haptics is quite a complex sense and apart from force, kinesthetic and mechanical tactile receptors it also includes receptors for detecting temperature, pain and various chemical stimuli. This complexity of the haptic sense is a strong motivation for developing a standard framework that describes haptic interaction. The primary intention of the framework is to provide a tool that allows useful guidelines on haptic interaction to be organized. Although haptic display is in its infancy, many researchers have performed ergonomic studies in this area and so many useful guidelines already exist. Unfortunately this information is fragmented and it is our intention to provide a unified body of knowledge for people working in this domain.

The primary aim of this paper then, is to present a framework for haptic interaction and discuss how it can be used as a tool to assist with the design of haptic interfaces. In particular we will discuss the role of the framework during analysis, design and evaluation of interfaces.

This framework, which is known as the GOTHI model (Carter, van Erp, et. al., 2005) was an outcome from the GOTHI-05 workshop (Guidelines On Tactile and Haptic Interactions, October 2005). This workshop brought researchers together who were interested in developing a collection of ergonomic guidelines and a framework for tactile and haptic interaction. The inaugural meeting of ISO TC159/SC4/WG9 committee further refined this initial framework and adopted it as the basis for structuring its new series of ISO standards on 
tactile and haptic interactions. The model itself will be elaborated in ISO 9241-910 Framework for Tactile and Haptic Interactions.

\section{THE BREADTH OF TACTILE AND HAPTIC INTERACTION}

Although haptic interaction is a relatively new domain, some interesting applications have been developed. For example, haptics has been used to display soil properties such as density, cohesion and angle of internal friction by allowing the user to move a simulated plough blade through various sandy soils (Green and Salsibury, 1998). Some of the more familiar applications of tactile/haptic interactions include: robotics, specialized control situations, and enhancing accessibility. However, there is a much wider set of applications.

Tactile and haptic interactions can go beyond providing alternate methods of accessing basic functionalities provided by other modalities. For example, visually impaired users can use a haptic display to feel the global structure of a painting and to identify different colors in the painting that can be represented by different textures (Sjöström, 1997).

Haptic widgets have been designed to assist navigation or targeting (Eberhardt et al., 1997; Miller, 1998) and force feedback has been used to provide additional spatial structure in the user interface (Miller, 1998). Orthogonal 3D haptic models have been used for: interactions with CAD models (Larsson, 1997), electronics test consoles (Davidson, 1996), anatomical organs (Mor et al., 1996), helping sculptors (Snibbe et al., 1998), and simulating LegoTM bricks (Young et al., 1997). 3D haptic spaces have also been used for scientific visualization in diverse domains such as: oil exploration (Nesbitt et al., 1997), mineral exploration (Veldkamp et al., 1998), representing magnetic fields (Fritz \& Barner, 1996), displaying fluid flow fields (Nesbitt et. al., 2001), gravity-wells (Oakley et al., 2000) and molecular docking studies (Brooks et. al., 1990).

The wide variety of research into applications of tactile/haptic interactions has also led to a wide variety of ergonomic guidelines for the design of tactile/haptic interactions (van Erp, 2002; Oakely et. al., 2002; Hale \& Stanney, 2004; Bresciani et. al., 2005; Carter \& Fourney, 2005; Fourney \& Carter, 2005; Nesbitt, 2005b). Additionally, various models of tactile/haptic interactions have been proposed (Popescu et. al., 2002; Carter, 2005; Nesbitt, 2005a). The haptic interaction model described in this paper draws heavily upon this earlier work.

\section{THE GOTHI MODEL OF THE TACTILE/HAPTIC DESIGN SPACE}

The Guidance on Tactile and Haptic Interaction, GOTHI05, workshop of invited experts started the process of transforming research-generated knowledge into a set of guidelines suitable for international standardization. Discussions at the workshop also highlighted the need for international standardization, when the various experts agreed to disagree on the definitions of a variety of fundamental terms, including tactile and haptic. The main achievement of GOTHI-05 was a model for organizing knowledge and guidance relating to tactile/haptic interaction (regardless of how one distinguishes between tactile and haptic).

The GOTHI model (Carter, van Erp, et. al., 2005) is intended to provide a number of different viewpoints for designers of haptic interaction. Not all designers are expected to approach haptic interaction from the same perspective and although some perspectives are complementary or overlap we intend to cover a range of design approaches that will allow better access to the guidelines organized within the framework.

The GOTHI model is hierarchical, and at the initial model was segmented to address the following key design issues: tactile/haptic inputs, outputs, and/or combinations; attributes of tactile/haptic for encoding of information; content-specific encoding; interaction tasks and interaction techniques. Further consideration of the initial GOTHI model led the ISO TC159/SC4/WG9 committee to expand the scope (ISO, 2006) and include six main dimensions. These dimensions are discussed in more detail below and include:

1. Applicability of tactile/haptic interactions

2. Tactile/haptic inputs and outputs

3. Attributes for tactile/haptic encoding of information

4. Layout of tactile/haptic objects

5. Interaction tasks

6. Interaction techniques

While there is a variety of specific research on the design of specific devices, further work is needed to understand how this research can be generalized into useful design principles of a wider range of devices, including devices that have not yet been contemplated. Therefore, the framework focuses on users and does not deal with specific haptic interface devices. Much further research is needed before guidance on haptic devices is ready for standardization.

\section{Applicability of tactile and haptic interactions}

Because many forms of tactile and haptic interaction are still relatively novel, a key concern for interface designers is when is tactile and haptic display applicable to their problem domain. The intention of this part of the GOTHI model is to give guidance to designers about what type of interaction is appropriate and when and how it might be used.

Of course some forms of tactile/haptic interaction are quite common in current interfaces. Indeed most users use tactile / haptic interactions as their main input mechanism, via keyboards and mice. Keyboards, mice, and various types of pointing devices are already covered by the ISO 9241-400 series of standards (ISO, 2005) that deal with physical input devices. Typical interactions between humans, keyboards and mice make use of only a small portion of all possible human computer tactile and haptic interactions. This has resulted in a notable lack of recognition of tactile or haptic interactions in the ISO 14915-3 (ISO, 2002) standard which deals with "media selection and combination" only in terms of audio and visual media. By contrast, this part of the GOTHI model (ISO 9241-910) deals with the application of the entire spectrum of available encodings, interaction tasks and interaction techniques possible with tactile and haptic display. 
Current views of how to apply tactile and haptic interaction tend to focus on simulating physical skills or providing a further channel of information for visually impaired users. For example, a traditional use of tactile output is for providing Braille output for the visually disabled. Haptic interactions have also become associated with physical simulations for activities such as driving, flying or medical training. There has also been a long association of haptic interfaces with applications involved remote operations in diverse fields such as mining and surgery.

However, the technology has also been used in more novel ways in computer games, scientific visualization and to enhance traditional graphical user interfaces. It is through these more general audience interfaces that tactile and haptic interactions stand the best chance of moving from the laboratory to the main stream of computing. ISO 9241-910 will provide guidance on the applicability of various types of tactile / haptic interactions to a range of user tasks.

Developers need to have a better understanding of how and where tactile/haptic interactions can be used in user interfaces, and especially how their use can improve usability for different groups of users. Thus the focus here is very much on the traditional user interface model that focuses on tasks and goals.

\section{Tactile/haptic inputs and outputs}

This section of the framework allows designers to approach the analysis and design of the interface by considering the types of haptic and tactile input and output that is available. It also includes considerations of both the uni-modal and multi-modal uses of tactile / haptic interaction. Since haptic displays are multi-modal in their own right, this also covers the use of multiple tactile devices.

It recognizes that while most other modalities are primarily used for inputs or for outputs, tactile/haptic interactions may be most effective when used for both inputs and outputs. The haptic system usually initiates action to stimulate sensory perception of the world. For example, to feel the hardness of a surface a person might tap on the surface. In this example, the motor system must initiate the sensory reception. That is, output is required to generate sensory input. Therefore haptic display devices are not passive displays, as is often the case with auditory and visual displays, but instead require an element of interaction from the user.

Dealing with this requires consideration of the effects of various combinations of input and output. For example vibration may mask other haptic outputs. There may also be sensory interactions between other modalities.

This section of the model also considers the need to support intentional individualization of individual tactile/haptic attributes to meet the capabilities and perceptions of individual users.

Developers need to consider how tactile/haptic interactions can be used with or without other modalities for different types of users. This consideration should lead to high level design decisions that will provide a basis for the further decisions identified by the remaining sections of this model.

\section{Attributes for tactile/haptic encoding of information}

Tactile/haptic encoding of information involves both the selection of tactile/haptic attributes to use for encoding information and additional concerns of how the type of information to be encoded relates to these attributes. For example, "Is it more appropriate to use friction or hardness to encode ordered categories of data?"

The tactile/haptic design space includes a range of spatial, temporal, and sensory attributes that may be used to encode information. For example: force, shape, size, friction, texture, mass/weight, hardness/softness, temperature, orientation, location, vibration, duration, motion, and deformation. This section discusses ergonomic issues relating to each attribute and their combinations.

The use of attributes for encoding requires an understanding of the user's capacity to perceive the intended encoding. For example, to allow a user to distinguish between two forces requires about a 7\% difference between them (Srinivasan \& Basdogan, 1997).

Different types of content with different needs include: textual data; graphical data (maps, pictures, figures/charts, textures, animations); longitudinal data, subjective data; statistical data and interface controls.

Developers need to consider a variety of issues in selecting the set of attributes to use to encode different types of information. This includes: the capacity of the attribute to encode the information to the desired precision, the user's expectations and experiences with other encodings, the interaction (interference or reinforcement) of combinations of attributes, and the type of content to be encoded. Issues of affordance and metaphor also must be considered. This part of the model can help developers by identifying these and other related issues that should be considered in designing tactile/haptic encodings.

\section{Layout of tactile/haptic objects}

A particular concern of designers, especially where haptic display is seen as an alternative to visual display, is how to make use of space and then layout structures within the interface space. Traditional visual displays are two dimensional, although new technologies are incorporating three dimensional spaces. While sound actually has many dimensions, the temporal dimension so predominates that, spatially at least, sound is usually designed in a single dimension (That is, all the sound generated from a single point in space). By contrast most previous uses of tactile/haptic interaction have utilized a three dimensional display space. This provides a variety of challenges for the designer in determining the layout of tactile/haptic objects. Whatever, the design developers need to adopt a consistent layout strategy for all tactile/haptic objects.

While haptic space is often three dimensional, not all tactile/haptic devices can utilize all three dimensions. For example, individual pins on a Braille display are laid out in two dimensions, while their height makes use of the third dimension to present information. 
Layout also depends on the conceptual framework within which individual objects are expected to exist. Thus, a real world framework would expect three dimensional layout, while a representation of a visual display would expect a two dimensional layout.

One specialized area of tactile/haptic display is the representation of spatial structures. The haptic sense like the visual sense is organized around a spatial map and this contrasts with the pitch-based organization of the auditory sense (Sekuler \& Blake, 1990, p. 314). Thus, the haptic sense can provide important cues about spatial structure, which are particularly important in interfaces for visually impaired users.

Developers need to adopt a consistent layout strategy for all tactile/haptic objects. Furthermore the design of a haptic space can build on ideas applied in the design of visual space. For example, space can be discrete or continuous and can even be designed so that the spatial map is distorted in some way, mush as space is distorted in visual displays incorporating a fish-eye view. This section of the framework is intended to provide guidance for such design issues.

\section{Interaction tasks}

Given the multi-dimensional space involved, it is important to recognize that there are a large number of different interaction tasks that a user might attempt. These interaction tasks can be classified as navigation tasks, selection tasks, or manipulation tasks.

Navigation tasks include: browsing / wayfinding; exploring the structure of the environment; exploring an object; targeting (going directly to a target object); using a search function, zooming the scale of the presentation, and reorienting by changing coordinates of the haptic space.

Selection tasks include: object selection; group selection (for a defined group); space selection (user defined portion of total space); and selection of system properties.

Manipulation tasks include: function activation, creation and deletion of objects and groups; getting information which may be objective or subjective in nature; modifying information (attributes \& relationships); and managing alternatives for individualization or personalization of the system.

Developers need to refer back to their problem domain and to be able to map interaction tasks to the needs of both application tasks and of users. Different users or situations may suit different methods of accomplishing the same general task. Thus, selecting to support only the minimal subset of interaction tasks may lead to usability/accessibility issues.

\section{Interaction techniques}

Interaction techniques deal with physical actions required of the user in order to accomplish various interaction tasks. Because of the many different types of physical touching, there are many more actions potentially involved in tactile/haptic interactions than are involved with traditional "direct manipulation" interfaces involving a mouse and a visual display. There are five main types of interaction techniques that impact on the design: moving relative to an object, moving an object, possessing an object, touching an object, and gesturing.

Moving relative to the object includes: tracking (moving to / from / with / by the object), tracing (moving across / around / along the surface of the object), entering the object, and pointing at an object.

Moving the object includes: dragging, pushing / pulling, displacing the object (shaking / tilting / twisting/ rotating), and directing object motion.

Because of the multi-dimensional nature of tactile/haptic layouts, it is possible that different coordinates, directions, and speeds may produce different results for each of these types of touching.

Possessing the object includes: grabbing / grasping, holding / gripping (e.g. continued grabbing/grasping), and releasing. Because of the interactive nature of tactile interfaces the strength, direction, and speed of executing these tasks may be significant.

Touching the object includes: tapping / hitting, pressing / squeezing / stretching, and rubbing the object. Again, attributes such as location, direction, force, and timing may all be significant.

While gesturing can be performed without physical contact, it is relevant when used with some tactile devices, such as data gloves.

Developers need to develop a clear semantics of how each of these techniques is used and interpreted.

\section{MOVING FORWARD WITH THIS MODEL}

The GOTHI model is the first model to identify the many dimensions of tactile/haptic output encodings and of tactile/haptic interaction tasks and techniques. This paper explains why each of the main components of this model is relevant to the design of interfaces making use of tactile/haptic interaction and identifies many of the aspects/attributes to be considered within each of these component areas.

The framework described above is intended to help developers and designers of interfaces incorporating tactile and haptic interaction by supporting both the analysis of problem domains and also the subsequent design of solutions involving haptic interaction. The framework (which will be published as ISO 9241-910) also provides categories for both collation of existing guidelines and further evaluation of the many aspect of tactile and haptic interaction. Indeed the intention of the ISO TC159/SC4/WG9 committee is that this framework will be used to structure guidelines (ISO 9241-920). The first two major products of ISO TC159/SC4/WG9 will be: ISO 9241910 and ISO 9241-920.

\section{ISO 9241-910 Framework for tactile / haptic interactions}

ISO 9241-910 will provide a detailed explanation of the model discussed above and the definitions used for the 900 series. This model will identify the various dimensions and properties of tactile/haptic interactions, based on in the GOTHI model. ISO 9241-910 will also describe in more detail 
how this model can be used to analyze, design, and evaluate interfaces that make use of tactile/haptic interactions. Work on this part will start in 2006-2007.

In particular this model can be used by developers to ensure that their analyses and designs have fully considered the possibilities and constraints of tactile/haptic interactions. The definitions will also help developers, researchers, and even end users to ensure that they share a common language.

\section{ISO 9241-920 Guidance on tactile / haptic interactions}

ISO 9241-920 provides detailed technology independent guidelines organized based on this model. ISO TC159/SC4/ WG9 has evolved these guidelines through a number of working drafts and expects to send then out for their first international ballot by early 2007. A companion paper (Fourney and Carter, 2006) provides further discussion of ISO 9241-920 and other parts of the ISO 9241-900 series.

Understanding the model and definitions from ISO 9241910 will help developers to find the appropriate ergonomic guidance in ISO 9241-920 to support design and evaluation of tactile/haptic interactions.

\section{REFERENCES}

Bresciani, J.-P., Drewing, K., Zopf, R., Wimperis, A., Lovell, G., Giachristsis, C., Roberts, R., Hesse, C., Helbig, H., Lange, C., Vitello, M., Bouyer, G., Maury, V., Kheddar, A., Bracewell, M., Wing, A., \& Ernst, M. (2005). System specifications design guidelines. Deliverable D5.7-Extended 2, TOUCH-HAPSYS Consortium.

Brooks, F., Ouh-Young, M., Batter, J., \& Kilpatrick, P. (1990). "Project GROPE - Haptic displays for scientific visualization." SIGGRAPH 1990, 177-185.

Carter, J. (2005). A Tactile/Haptic Interface Object Reference Model. In Proceedings of Guidelines On Tactile and Haptic Interactions (GOTHI'05) Conference, Saskatoon, Saskatchewan, Canada, 6 pages. http://userlab.usask.ca/Carter-Model.pdf

Carter, J., \& Fourney, D. (2005). Research based tactile and haptic interaction guidelines. In Proceedings of Guidelines On Tactile and Haptic Interactions (GOTHI'05) Conference, Saskatoon, Saskatchewan, Canada, 9 pages. http://userlab.usask.ca/research.pdf

Carter, J., van Erp, J., Fourney, D., Fukuzumi, S., Gardner, J.A., Horiuchi, Y., Jansson, G., Jürgensen, H., Kadefors, R., Kobayashi, T., Kwok, M.G., Miyagi, M., and Nesbitt, K.V. (2005). The GOTHI model of tactile and haptic interaction. In Proceedings of GOTHI-05 Guidelines on Tactile and Haptic Interactions (pp. 93-95). Available at: http://userlab.usask.ca/GOTHI/ Complete Proceedings of GOTHI-05.pdf

Davidson, S. (1996). "A Haptic Process Architecture using the PHANTOM as an I/O Device in a Virtual Electronics Trainer". The First PHANToM User's Group Workshop, pp.35-38, Cambridge, Massachusetts, USA, MIT.

Eberhardt, S., Neverov, M., West, T., \& Sanders, C. (1997). "Force reflection for WIMPs: A button acquisition experiment". ASME Dynamic Systems and Control Division. DSC-Vol. 61.

Fourney, D., \& Carter, J. (2005). Initiating guidance on tactile and haptic interactions. In Proceedings of Guidelines On Tactile and Haptic Interactions (GOTHI'05) Conference, Saskatoon, Saskatchewan, Canada, 9 pages. http://userlab.usask.ca/Fourney-Guidance.pdf

Fourney, D., \& Carter, J. (2006). Existing and future guidance on tactile and haptic interactions, Proceedings of the $50^{\text {th }}$ Annual Meeting of the Human Factors and Ergonomics Society.

Friedes, D. (1974). Human information processing and sensory modality: Cross-modal functions, information complexity, memory and deficit. Psychological Bulletin, 81(5), 284-310.

Fritz, J., \& Barner K. (1996). "Haptic scientific visualization". The First PHANToM User's Group Workshop, pp.70-73, Cambridge, Massachusetts, USA, MIT.
Green, D., F. and J. K. Salsibury (1998). "Soil Simulation with a PHANToM". The Third PHANToM User's Group Workshop, Cambridge, Massachusett, USA, MIT.

Hale, K., \& Stanney, K. (2004). Deriving haptic design guidelines from human physiological, psychophysical, and neurological foundations. IEEE Computer Graphics and Applications, 24(2), 33-39.

ISO. (2002). ISO 14915-3:2002 Software ergonomics for multimedia user interfaces - Part 3: Media selection and combination. International Organization for Standardization, Geneva, Switzerland.

ISO. (2005). ISO CD 9241-400:2005: Ergonomics of human-system interaction - Physical input devices - Part 400: Guiding principles, introduction and general design requirements. International Organization for Standardization, Geneva, Switzerland.

ISO. (2006). ISO 9241- 920 Ergonomics of human system interaction - Guidance on tactile and haptic interactions, 1st working draft. ISO TC159/SC4/WG9 Document N012. International Organization for Standardization, Geneva, Switzerland.

Larsson, P. (1997). "Adding Force Feedback to Interactive Visual Simulations using Oxygen". The Second PHANToM User's Group Workshop, pp.6062, Cambridge, Massachusetts, USA, MIT.

Miller, T. (1998). "Implementation Issues in Adding Force Feedback to the X Desktop". The Third PHANToM User's Group Workshop, pp.50-53, Cambridge, Massachusetts, USA, MIT.

Mor, A., Gibson, S., \& Samosky, J. (1996). "Interacting with 3-Dimensional Medical Data Haptic Feedback for Surgical Simulation". The First PHANToM User's Group Workshop, pp.83-86, Cambridge, Massachusetts, USA, MIT.

Nesbitt, K., Orenstein, B., Gallimore, R., \& McLaughlin, J. (1997). "The Haptic Workbench applied to Petroleum 3D Seismic Interpretation". The Second PHANToM User's Group Workshop, pp.63-67, Cambridge, Massachusetts, USA, MIT.

Nesbitt, K., Gallimore, R., \& Orenstein, B. (2001). "Using Force Feedback for Multi-sensory Display". 2nd Australasian User Interface Conference (AUIC 2001), Gold Coast, Queensland, Australia, IEEE Computer Society.

Nesbitt, K. (2005a). A structured framework to support the designers of haptic, visual and auditory displays. In Proceedings of Guidelines On Tactile and Haptic Interactions (GOTHI'05) Conference, Saskatoon, Saskatchewan, Canada, 11 pages. http://userlab.usask.ca/Nesbitt-Model.pdf

Nesbitt, K. (2005b). Structured guidelines to support the design of haptic displays. In Proceedings of Guidelines On Tactile and Haptic Interactions (GOTHI'05) Conference, Saskatoon, Saskatchewan, Canada, 10 pages. http://userlab.usask.ca/Nesbitt-Guidelines.pdf

Oakley, I., McGee, M., Brewster, S., \& Gray, P. (2000). "Putting the feel in look and feel". ACM Computer Human Interaction 2000, Hague, Netherlands, ACM Press Addison-Wesley. 415-422.

Oakley, I., Adams, A., Brewster, S., and Gray, 2002, P. Guidelines for the design of haptic widgets. In Proceedings of BCS HCI 2002, London, UK, Springer, pp. 195-212.

Popescu, V., Burdea, G., \& Trefftz, H. (2002). Multimodal interaction modeling. In K. M. Stanney, (Ed.), Handbook of virtual environments: Design, implementation, and applications. Mahwah, NJ: Lawrence Erlbaum Associates.

Sekuler, R., \& R. Blake. (1990). Perception. New York: McGraw-Hill Publishing Company.

Sjöström, C. (1997). "The PHANToM for blind people". The Second PHANToM User's Group Workshop, pp.85-88, Cambridge, Massachusetts, USA, MIT.

Snibbe, S., Anderson, S., \& Verplank, B. (1998). "Springs and constraints for 3D drawing". The Third PHANToM User's Group Workshop, pp.59-62, Cambridge, Massachusetts, USA, MIT.

Srinivasan, M., \& Basdogan, C. (1997). Haptics in virtual environments: Taxonomy, research status, and challenges. Computer \& Graphics, 21(4), 393-404.

van Erp, J. (2002). Guidelines for the use of vibro-tactile displays in human computer interactions. In Proceedings of EuroHaptics 2002.

Veldkamp, P., Turner, G., Gunn, C., \& Stevenson, D. (1998). "Incorporating Haptics into Mining Industry Applications". The Third PHANToM User's Group Workshop, pp.80-84, Cambridge, Massachusetts, USA, MIT.

Young, P., Chen, T., Anderson, D., Yu, J., \& Nagata, S. (1997). "LEGOLAND: A Multi-Sensory Environment for Virtual Prototyping". The Second PHANToM User's Group Workshop, pp.56-59, Cambridge, Massachusetts, USA, MIT. 